\title{
Development of a Sterne-Based Complement Fixation Test to Monitor the Humoral Response Induced by Anthrax Vaccines
}

\author{
Rosanna Adone ${ }^{1}$, Michela Sali ${ }^{2}$, Massimiliano Francia ${ }^{1}$, Michela latarola ${ }^{3}$, \\ Adelia Donatiello ${ }^{3}$ and Antonio Fasanella ${ }^{3 *}$ \\ ${ }^{1}$ Istituto Superiore di Sanità, Rome, Italy, ${ }^{2}$ Università Cattolica del Sacro Cuore, Rome, Italy, ${ }^{3}$ Istituto Zooprofilattico \\ Sperimentale della Puglia e Basilicata, National Reference Centre for Anthrax, Foggia, Italy
}

Anthrax is a zoonotic disease caused by Bacillus anthracis spore-forming bacterium. Since it is primarily a disease of animals, the control in animals, and humans depend on the prevention in livestock, principally cattle, sheep, and goats. Most veterinary vaccines utilize the toxigenic, uncapsulated (pXO1+/pXO2-) $B$. anthracis strain $34 \mathrm{~F}_{2}$ which affords protection through the production of neutralizing antibodies directed to the toxin components Protective Antigen (PA), Lethal Factor (LF), and Edema Factor (EF).

OPEN ACCESS

Edited by: Bassam Hallis, Public Health England, UK

Reviewed by:

Shivani Agarwal,

Northwestern University, USA

Martin Eales Hugh-Jones, Louisiana State University, USA

*Correspondence: Antonio Fasanella a.fasanella@izsfg.it

Specialty section: This article was submitted to Infectious Diseases, a section of the journal Frontiers in Microbiology

Received: 27 July 2015 Accepted: 11 January 2016 Published: 28 January 2016

Citation:

Adone R, Sali M, Francia M latarola $M$, Donatiello $A$ and Fasanella A (2016) Development of a Sterne-Based Complement Fixation Test to Monitor the Humoral

Response Induced by Anthrax Vaccines. Front. Microbiol. 7:19. doi: 10.3389/fmicb.2016.00019
The titration of specific antibodies in sera of vaccinated animals is crucial to evaluate the efficacy of the vaccination and to obtain epidemiological information for an effective anthrax surveillance. In this study, we developed a Sterne-based Complement Fixation Test (CFT) to detect specific antibodies induced in animals vaccinated with Sterne $34 \mathrm{~F}_{2}$. We assessed its efficacy in laboratory animals and under field conditions by monitoring the humoral response induced by vaccination in cattle. The results indicated that the Sterne-based CFT is able to correctly identify vaccinated animals. It proved to be a very sensitive and specific test. Moreover, the Sterne-based CFT offers many benefits with regard to costs, standardization and reproducibility of the assay procedure.

Keywords: anthrax, serology, complement fixation test, antibody response, vaccination

\section{INTRODUCTION}

Anthrax is a zoonotic disease with an almost worldwide distribution, with prevalence in tropical and sub-tropical countries (Biswas et al., 2011). The causative agent is Bacillus anthracis, a Grampositive, aerobic, spore-forming bacterium. It is primarily a disease of herbivores; ruminants such as cattle, goats, and sheep are the most susceptible animals (World Health Organization [WHO], 2008). Animals become infected thorough contact with soil contaminated with persistent bacterial spores. Humans are susceptible to infection through contact with infected animals or animal products contaminated with anthrax spores. Depending of the route of the exposure, human anthrax infection can take three forms: cutaneous, gastro-intestinal, and inhalational anthrax. The majority of reported cases are cutaneous (95\%), especially in developing countries whose main source of income is farming (Ghosh and Goel, 2012).

Bacillus anthracis has two principal virulence factors, the toxin complex, and the poly$\gamma$-D-glutamic acid capsule, coded by plasmids pXO1 and pXO2, respectively. The capsule 
protects the bacterium from phagocytosis while the toxin complex consists of three synergistically acting proteins, Protective Antigen (PA), Lethal Factor (LF), and Edema Factor (EF), produced during the log phase of growth. The interaction of these proteins forms the lethal and edema toxins, responsible for cytotoxic effects (Collier and Young, 2003; World Health Organization [WHO], 2008). The protection against anthrax mainly depends on the host's humoral response to the toxin components (Turnbull et al., 1988; Pitt et al., 2001; Friedlander et al., 2002). The PA is a key element: the anti-PA antibodies are not, in themselves, a guarantee of protected status, but they must be in the blood of animal or human for the subject to be protected (Ivins and Welkos, 1988; Ivins et al., 1990; Little et al., 1997).

Since anthrax is primarily a disease of animals, its control in animals and humans depends on the prevention in livestock, principally cattle, sheep, and goats. Most anthrax vaccines for animals utilize the $B$. anthracis strain $34 \mathrm{~F}_{2}$, developed by Max Sterne in 1937 (Sterne, 1939) which lacks genes for capsule formation but still produces the toxin (pXO1+/pXO2-), responsible for the induction of protective antibodies. The protective effect of a single dose of this vaccine is said to last for 9 to 12 months so annual boosters are recommended in endemic areas (Sterne, 1939). Also, a single dose of Sterne vaccine may not be sufficient to ensure protective immunity in the animal to last for a year, and more than one initial dose of the Sterne vaccine may be necessary (Turnbull et al., 2004; Mongoh et al., 2008; World Health Organization [WHO], 2008). However, very few studies focusing on immunological characterization have been conducted with cattle and goats (Dipti et al., 2013; Roy et al., 2013).

Serological methods, which have not a prominent role in diagnosis, are very useful epidemiological and research tools (Turnbull et al., 1992; Quinn et al., 2004) since they enable to monitor the humoral response following vaccination or naturally acquired infection. The titration of specific antibodies in sera of vaccinated animals is crucial to evaluate the efficacy of the vaccination and to obtain epidemiological information in areas where the disease is endemic.

Currently, the ELISA is considered the most effective serological method; however, it utilizes purified toxin antigens PA and LF whose preparation is expensive and lacks of a good standardization in regard to purity, composition of antigens and preparation procedure. Moreover, reference standard serum is not available.

The Complement Fixation Test (CFT) is a serological method prescribed as individual confirmatory test in many infectious diseases. It is able to detect specific antibodies in animal and human serum samples and may detect incomplete antibodies. The antigen preparation, mainly consisting in an inactivated bacterial suspension, is not expensive and can be easily standardized.

The purpose of this study was to develop a Sterne-based CFT able to detect anti-anthrax antibodies induced in animals vaccinated with the Sterne $34 \mathrm{~F}_{2}$ vaccine. We assessed the efficacy of our method in laboratory animals and under field conditions by monitoring the humoral response induced in cattle by vaccination with Sterne $34 \mathrm{~F}_{2}$.

\section{MATERIALS AND METHODS}

\section{Bacterial Strains and Growth Conditions}

The $B$. anthracis strain $34 \mathrm{~F}_{2}$, used for the preparation of the veterinary vaccine, and the virulent strain B. anthracis A0843 (Fasanella et al., 2001) were supplied by the National Reference Centre for Anthrax (Ce.R.N.A.) of the Istituto Zooprofilattico Sperimentale of Puglia and Basilicata (Foggia, Italy). This Institute is charged with the production of the Sterne $34 \mathrm{~F}_{2}$ vaccine, routinely used for immunization of susceptible animal species in Italy (Italian DM 7-7-1992). Both strains were cultured at $37^{\circ} \mathrm{C}$ on agar tryptose soy agar supplemented with $5 \%$ of equine serum (TSA/S).

\section{Animals}

In this study the ability of the Sterne-based CFT to detect the antibody response induced by vaccination with Sterne $34 \mathrm{~F}_{2}$ was evaluated in rabbits and cattle.

New Zealand White (NZW) rabbits, weighting $1.2-1.5 \mathrm{~kg}$, provided by Harlan Laboratories srl (Udine, Italy) were individually housed in barrier housing with filtered inflow air in a restricted-access room and under pathogen-limited conditions. Experiments were conducted in accordance with the current European Legislation (Directive 86/609/EEC) relating the welfare of animals involved in experiments.

Breed Limousine female cattle, 10-12 months of age, belonging to a farm in Basilicata, a southern region of Italy where anthrax is enzootic, were used.

\section{Experimental Design}

Thirty-eight NZW rabbits were vaccinated subcutaneously (s.c.) two times, at 15 days interval, with $1 \mathrm{ml} /$ each containing $1.2 \times 10^{7}$ live spores of Sterne $34 \mathrm{~F}_{2}$ vaccine. Blood samples were collected from all rabbits prior to vaccination (day 0), 15 days after the first vaccination (15 days post-vaccination, dpv) and 15 days after the second vaccination (30 dpv). Nine unvaccinated rabbits were kept as controls.

On the same day of the last sampling, all rabbits, vaccinated and controls were challenged with $200 \mathrm{LD}_{50}$ of $B$. anthracis virulent strain A0843 to evaluate the protective activity of the vaccine.

Forty-six female cattle were vaccinated subcutaneously with $1 \mathrm{ml} /$ each containing $1.2 \times 10^{7}$ live spores of Sterne $34 \mathrm{~F}_{2}$ vaccine, as prescribed by the Italian DM 7-7-1992. Ten, unvaccinated female cattle belonging to the same farm were kept as controls.

Blood samples were collected prior vaccination (day 0) and then at 12,30 , and $150 \mathrm{dpv}$.

After incubation at $37^{\circ} \mathrm{C}$ for $30 \mathrm{~min}$ to clot, serum samples were collected by centrifugation and stored at $-20^{\circ} \mathrm{C}$ until use. All sera were tested by the Sterne-based CFT.

\section{Complement Fixation Test: Principle of the Method}

The CFT consists of two stages: in the first stage, specific antigen and test serum are mixed with guinea-pig serum which contains the complement. If specific antibodies are present in the test 
serum, the complement combines with the antigen-antibody complex and cannot react in the second stage of the reaction. In the second stage, sheep erythrocytes previously sensitized with rabbit serum anti-sheep erythrocytes (hemolytic system), are added. If the complement has been fixed in the first stage, no hemolysis will occur (positive reaction). On the contrary, if the serum test doesn't contain specific antibodies, the complement, not consumed, can combine with the hemolytic system causing the lysis of sheep erythrocytes (negative reaction).

The CFT required the following reagents: (i) lyophilized guinea-pig complement (serum of healthy guinea pigs); (ii) hemolysin stock solution (monoclonal antibodies against sheep erythrocytes), (iii) sheep erythrocytes $\left(54 \times 10^{7}\right.$ cells $\left./ \mathrm{ml}\right)$; (iv) bovine, hyperimmune serum containing anti-anthrax antibodies (positive control); (v) pool of sera from healthy, unvaccinated cattle (negative control).

For this study, sheep erythrocytes, hemolysin, and complement were supplied by Emozoo Snc of Ripabelli G. \& C. (Siena, Italy). Positive and negative controls, previously evaluated by ELISA (Fasanella et al., 2007), were provided by the Ce.R.N.A.

The Veronal calcium-magnesium buffer, $\mathrm{pH} 7.2 \pm 0.1$ (Lonza, Walkersville, Inc., USA), added with $0.1 \%$ of bovine serum albumin (VBA), was used as diluent.

\section{Sterne Antigen Preparation and Titration for Use in CFT}

The Sterne antigen for CFT was prepared as follows: single colonies of $B$. anthracis strain $34 \mathrm{~F}_{2}$ were cultured for $24 \mathrm{~h}$ at $37^{\circ} \mathrm{C}$ on TSA/S plates and then on Roux flasks for propagation. After incubation, bacteria were harvested with physiological saline $(\mathrm{pH}$ 7.2), inspected for purity by Gram staining and washed twice by centrifugation at $1.430 \mathrm{~g}$ for $15 \mathrm{~min}$. For the inactivation, the bacterial suspension was added with $3 \%$ of formalin saline and incubated overnight at $37^{\circ} \mathrm{C}$. After inactivation checking, the suspension was centrifuged as above and the pellet was suspended in $0.5 \%$ of formalin saline for storage until use.

For use in the CFT, the Sterne antigen was titrated as prescribed by the OIE Manual of Diagnostic Tests for diagnosis of brucellosis (Office International des Epizooties [OIE], 2008). Briefly, doubling dilutions of the antigen, each supplemented with 5\% of Fetal Calf Serum (FCS, OXOID), were tested in CFT to establish the optimal concentration which was able to: (i) react positively with the positive control; (ii) do not react with the negative control; (iii) do not show anticomplementary activity. The anticomplementary activity occurs when the antigen fixes the complement in absence of the antigen-antibody complex, thus giving false positive reactions.

The optical density of the optimal antigen concentration, measured at $490 \mathrm{~nm}$, gave a value of 0.2 .

\section{Sterne-Based CFT on Rabbit and Bovine Serum Samples}

Before the CFT, all sera were diluted 1:2 in VBA and incubated for $30 \mathrm{~min}$ at the appropriate temperature to inactivate the native complement $\left(58^{\circ} \mathrm{C}\right.$ for cattle, $62^{\circ} \mathrm{C}$ for rabbits). The Sternebased CFT was performed as previously described (Adone and Ciuchini, 1999; Adone et al., 2008). Briefly, in 96-well roundbottom plates $25 \mu \mathrm{l}$ of each serum was serially diluted in VBA from 1:2 to $1: 128$. Then, $25 \mu \mathrm{l}$ of antigen and $25 \mu \mathrm{l}$ of complement, at working dilution, were added and plates were incubated at $37^{\circ} \mathrm{C}$ for $30 \mathrm{~min}$. After incubation, $25 \mu \mathrm{l}$ of sensitized erythrocytes were added to each well. After incubation as above, plates were centrifuged at $200 \mathrm{~g}$ for $5 \mathrm{~min}$ to allow any unlysated cells to deposit and the reaction was read over a diffused white light. The results were evaluated as follows: $100 \%$ hemolysis was considered as negative reaction while all reactions showing complete absence of hemolysis ( $0 \%$ ) or partial hemolysis 75,50 , or $25 \%$ were considered as positive. The titer of each serum was the highest dilution showing a positive reaction. Based on our previous experience (unpublished data), the serum dilution 1:2 showing $50 \%$ of hemolysis was taken as the reactivity threshold of the reaction.

\section{Sensitivity (SN) and Specificity (SP) of the Sterne-Based CFT}

The SN and SP of the Sterne-based CFT were determined, at different times, by categorizing the results into reactive and nonreactive by application of the reactivity threshold (titer 1:2). The SP of the assay was calculated as TN/(TN+FP), where $\mathrm{TN}=$ true negatives and $\mathrm{FP}=$ false positives. The $\mathrm{SN}$ of the assay was calculated as $\mathrm{TP} /(\mathrm{TP}+\mathrm{FN})$, where $\mathrm{TP}=$ true positives and $\mathrm{FN}=$ false negatives.

Sterne-vaccinated animals were considered as true positives, while unvaccinated controls and animals bled prior vaccination were considered as true negatives.

\section{Statistical Analysis}

Different percentage of serology performed at different time points was calculated by using Contingency tables for nonparametric data (Fisher's exact test). Results were considered significant at $p \leq 0.05$.

\section{RESULTS}

\section{Rabbit Sera CFT Titers}

The results of the Sterne-based CFT performed on sera collected from $38 \mathrm{NZW}$ rabbits vaccinated with Sterne $34 \mathrm{~F}_{2}\left(1.2 \times 10^{7}\right.$ live spores /each), are showed in Figure 1. As shown, none of serum samples from rabbits bled prior vaccination (dpv 0$)$ and from nine unvaccinated controls gave positive results. At 15 days after the first vaccination (15 dpv), 89\% (34/38) of rabbits weakly reacted in CFT, titers ranging from 1:2 (15/38), considered as the reactivity threshold of the reaction, to $1: 8(1 / 38)$. The majority of them, (18/38), gave a titer of $1: 4$. In contrast, at 15 days after the second vaccination (30 dpv), all rabbits (38/38) were seropositive with titers ranging from $1: 4(2 / 38)$ to $1: 64(4 / 38)$, with a prevalence of titer 1:16 (18/38). Five rabbits (5/38) titrated $1: 8$, and nine rabbits (9/38) gave a titer 1:32. All CFT titers were significantly over the reactivity threshold (Fisher's exact test $p<0.01)$. 


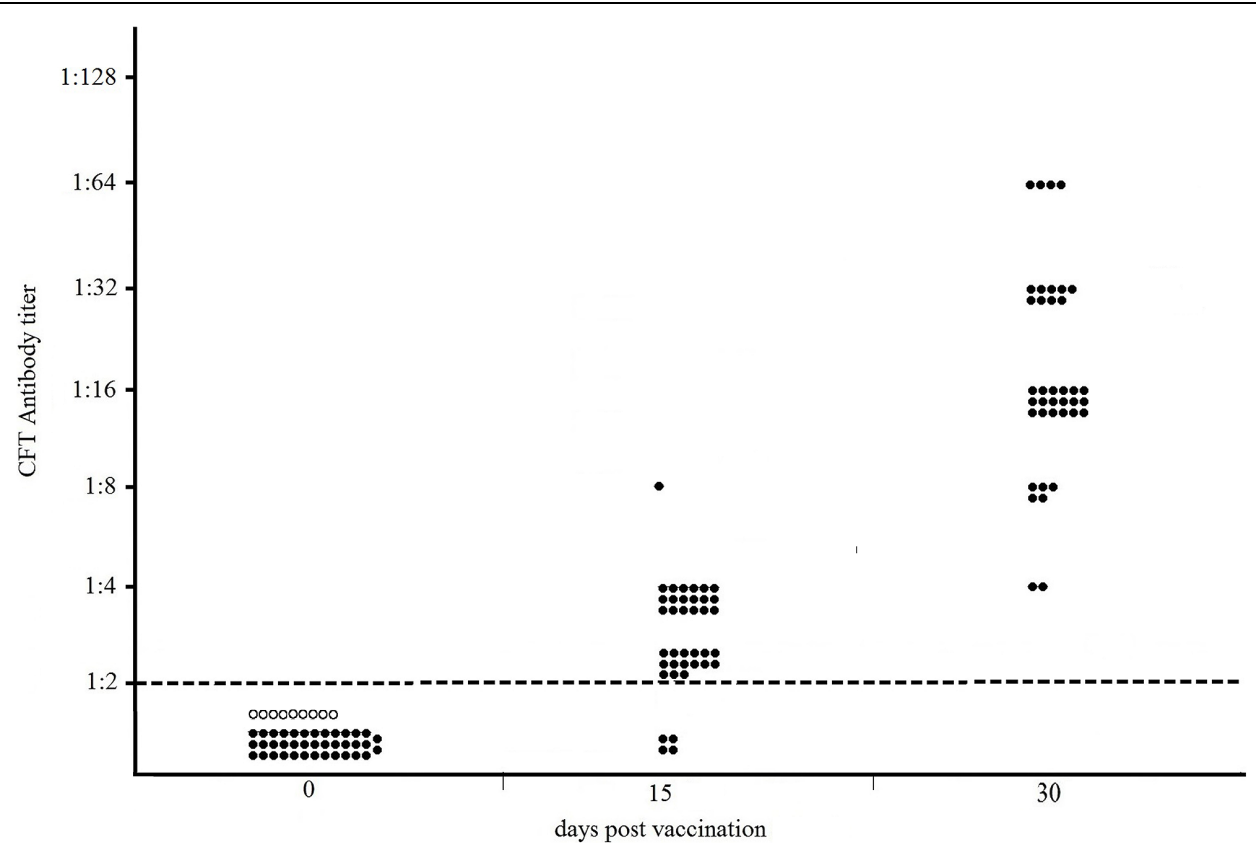

FIGURE 1 | Distribution of CFT titers of thirty-eight NZW rabbits vaccinated with $1.2 \times 10^{7}$ live spores of Sterne $34 F_{2} /$ each, measured by Sterne-based CFT prior to vaccination (dpv $\mathbf{0}$ ), at $\mathbf{1 5}$ and $\mathbf{3 0}$ days post vaccination. At $15 \mathrm{dpv}$, rabbits received the second dose of vaccine. Black and white circles represent the vaccinated and unvaccinated (controls) rabbits, respectively. The broken line indicates the reactivity threshold of the reaction (titer 1:2).

All seropositive rabbits survived to the challenge with the virulent strain while all controls died (data not shown).

\section{Bovine Sera CFT Titers}

The results of the Sterne-based CFT performed on sera collected from 46 cattle vaccinated with Sterne $34 \mathrm{~F}_{2}\left(1.2 \times 10^{7}\right.$ live spores /each), are presented in Figure 2. As shown, none of cattle bled prior to vaccination ( $\mathrm{dpv} 0$ ) or unvaccinated controls were seropositive, thus indicating $100 \%$ of specificity. At 12 days post vaccination, complement fixating antibodies to Sterne were detected in $72 \%$ of calves (33/46), titers ranging from 1:4 (16/46) to $1: 32(1 / 46)$. Eight cattle $(8 / 46)$ gave a titer $1: 8$ and the remaining eight cattle $(8 / 46)$ gave a titer $1: 16$. At $30 \mathrm{dpv}$, all cattle (46/46) were seropositive, titers significantly increasing from 1:16 $(1 / 46)$ to $1: 128(1 / 46)$. Most of them $(31 / 46)$ gave a titer $1: 32$, while 13/46 titrated 1:64. All CFT titers were significantly over the reactivity threshold. At $150 \mathrm{dpv}$, only 13\% (6/46) of calves were still seropositive (Fisher's exact test $p=0.02$ ) with decreased titers from 1:4 (3/46) to 1:16 (1/46). Two cattle (2/46) titrated 1:8. Six cattle $(6 / 46)$ gave a titer $1: 2$ corresponding to the reactivity threshold of the reaction.

\section{Kinetic of the Antibody Response Induced by Vaccination in Cattle}

As shown in Figure 2, at 12 days post vaccination, detectable antibodies to Sterne were present in $72 \%$ of cattle $(33 / 46)$. At $30 \mathrm{dpv}, 100 \%$ of cattle were seropositive, showing high and similar antibody titers to Sterne antigen. At $150 \mathrm{dpv}$, specific antibodies to Sterne were detected only in $13 \%$ of cattle.

\section{DISCUSSION}

The protective activity of anthrax vaccines mainly depends on their ability to elicit antibodies directed to toxin components (Little et al., 1997; Beedham et al., 2001; Reuveny et al., 2001; Kobiler et al., 2002); however, little is known on the effective duration of immunity, many studies demonstrating the decline of the specific antibody response and the need of different vaccination schedules to ensure protection. New, improved livestock vaccines should be developed able to induce high level of protective antibodies in a very short time and that could be administered with long-acting antibiotics during the anthrax outbreaks or in emergency (Welkos et al., 2001; Fasanella et al., 2008; World Health Organization [WHO], 2008).

For humans, licensed vaccines are composed by killed, cellfree suspensions, to avoid the risks due to the residual virulence of live strains. They are targeted at persons occupationally exposed to anthrax spores or involved in occupations related to defense.

In contrast, most veterinary vaccines are composed by live spores of attenuated (non-capsulated) B. anthracis strain $34 \mathrm{~F}_{2}$ (Sterne, 1939) suspended in saponin for enhancing immune response. The non-capsulated variants of $B$. anthracis may lose their immunogenicity when cultured, so their efficiency must be monitored (Sterne, 1939, 1959).

Serological tests, which have not a great diagnostic value, proved to be a very useful epidemiological and research tool to evaluate the seroconversion following vaccination or naturally acquired infection (Turnbull et al., 1992; Quinn et al., 2004). Demonstration of specific antibodies in sera of vaccinated animals is needed to obtain epidemiological information in 


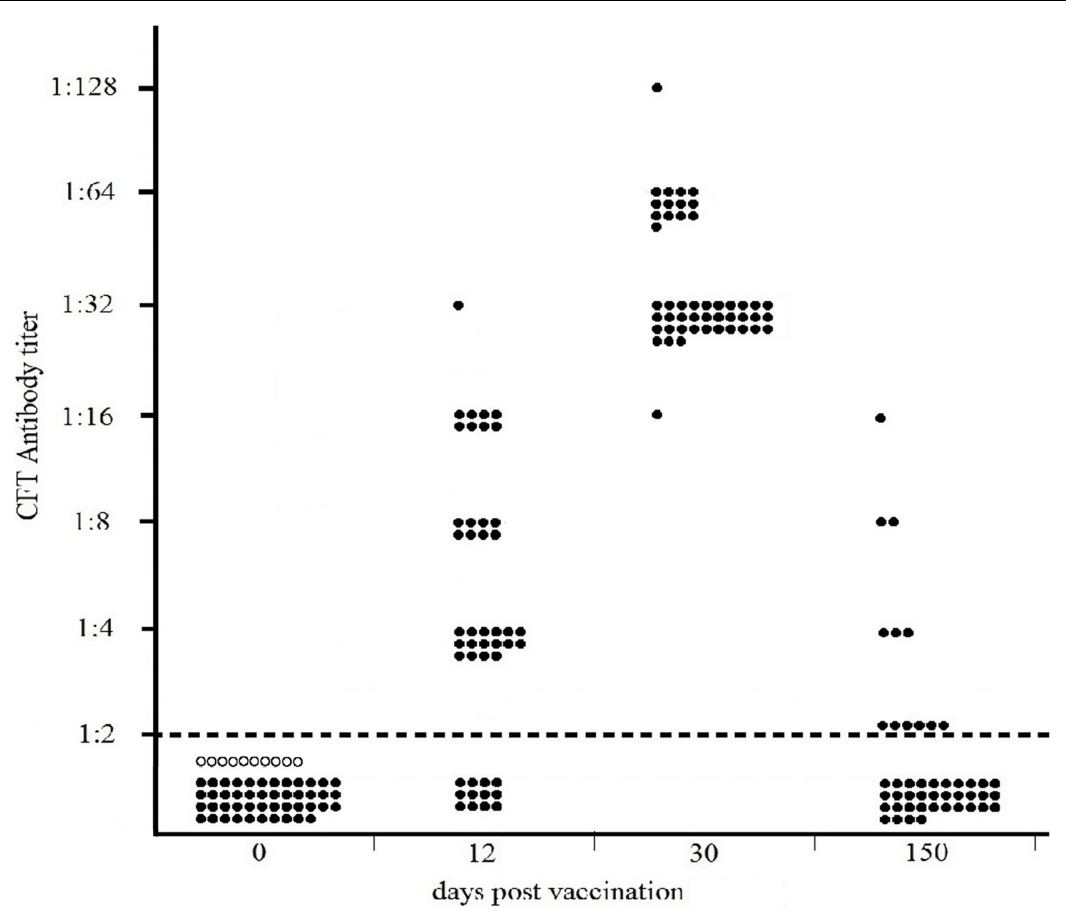

FIGURE 2 | Distribution of CFT titers of forty-six cattle vaccinated with $1.2 \times 10^{7}$ live spores of Sterne $34 F_{2} /$ each, measured by Sterne-based CFT prior to vaccination ( $\mathbf{d p v} \mathbf{0}$ ) and at 12, 30, and $\mathbf{1 5 0} \mathbf{d p v}$. Black and white circles represent the vaccinated and unvaccinated (controls) cattle, respectively. The broken line indicates the reactivity threshold of the reaction (titer 1:2).

areas where the disease is endemic and to evaluate the efficacy of the vaccination. In effect, there is still a general paucity of information related to the onset, kinetic, and magnitude of antibody response induced by vaccination in humans and animals.

Effective serological tests should be satisfactory in terms of sensitivity and specificity, not expensive, to avoid that their use is confined to a few specialist laboratories, and easy to standardize.

In this study, we assessed the suitability of a Sterne-based CFT as method for the detection of specific antibodies in laboratory and target animals vaccinated with Sterne $34 \mathrm{~F}_{2}$. We evaluated its specificity and sensitivity by testing unvaccinated and vaccinated animals, respectively, at different sampling times.

We prepared and titrated an inactivated Sterne suspension for use as antigen in CFT according to OIE indications and our CFT experience (Adone and Ciuchini, 1999; Adone et al., 2008). The addition of FCS increases the sensitivity of the reaction, reducing the number of false negative results as previously suggested (Cho, 1971).

The humoral response in NZW rabbits vaccinated with Sterne $34 \mathrm{~F}_{2}$ was evaluated according to the procedure used by Ce.R.N.A. to measure the potency of anthrax vaccines. For this purpose, rabbits were vaccinated twice, 15 days apart, with $1.2 \times 10^{7}$ live spores of Sterne $34 \mathrm{~F}_{2}$ and 15 days after the second vaccination all rabbits, vaccinated and controls, were challenged with $200 \mathrm{LD}_{50}$ of the virulent strain B. anthracis A0843. The antibody response was monitored prior to vaccination, 15 days after the first vaccination, and 15 days after the second vaccination, on the same day of the challenge. As shown in Figure 1, the Sternebased CFT was able to detect all vaccinated rabbits at $30 \mathrm{dpv}$, 15 days after the second vaccination. Most of seropositive rabbits gave complement fixating titers ranging from 1:16 to $1: 32$ thus significantly over the reactivity threshold. All seropositive rabbits survived to the challenge with the virulent strain while all controls died (data not shown) indicating that the humoral response measured by the Sterne-based CFT was protective. None of serum samples collected from rabbits prior vaccination or from unvaccinated controls gave positive results, thus indicating $100 \%$ of specificity (Figure 1).

Data obtained in rabbits indicated that a correlation between the complement fixating titers to Sterne and the protective activity afforded by the vaccine may be experimentally established. Based on our results, rabbits showing detectable complement fixating antibodies with titers significantly over the threshold of 1:2 were protected against anthrax infection.

Additional tests are in progress in our laboratory to confirm the suitability of the Sterne-based CFT as tool to measure the potency of anthrax vaccines in laboratory animals by evaluating antibody response in replacement of the challenge with virulent strains. The availability of methods alternative to experimental infection could avoid the need of containment equipment and facilities required to reduce the risks for personnel exposed to biosafety level 3 agents (Little et al., 2004). Moreover, it could minimize animal suffering, in agreement with principles for more ethical use of animals. 
In cattle, the Sterne-based CFT was able to detect vaccinated animals with a sensitivity ranging from $72 \%$ at $12 \mathrm{dpv}$ to $100 \%$ one month after the vaccination (Figure 2). At this time, complement fixating titers ranged from 1:16 to $1: 128$ with a prevalence of titer 1:32 thus significantly over the reactivity threshold. At $150 \mathrm{dpv}$, only 13\% of cattle were still seropositive, showing weak CFT titers. None of cattle bled prior vaccination or unvaccinated cattle gave positive results, thus confirming the high specificity of this test (Figure 2).

To evaluate the ability of the Sterne-based CFT to detect antibodies in other species, we tested serum samples from Sternevaccinated sheep, goats and horses: all vaccinated animals were identified, while proven uninfected or unvaccinated animals did not react (data not shown). Continuing validation is ongoing as samples with proven status and provenance become available.

As a serological method, the Sterne-based CFT could offer many benefits: (i) the reagents are not expensive and may be easily standardized: the optimal concentration of the antigen may be accurately determined and standardized. The other reagents are titrated according to classical procedures described by the OIE; (ii) the preparation of the antigen does not require particular growth conditions or safety measures since risks to personnel manipulating the Sterne $34 \mathrm{~F}_{2}$ are not greater than those posed by other category 2 organisms (Centers for Diseases Control and Prevention, Division of Foodborne, Bacterial and Mycotic Diseases, 2009); (iii) high-quality samples are not required, the anticomplementary reactions occurring rarely; (iv) the CFT is not animal species-specific: the same reagents are used to test sera from a variety of animal species and humans.

We did not test serum samples from anthrax-vaccinated people: however, since the procedure for detection of animal antibodies are identical to those used for human antibodies, we think that the Sterne-based CFT could be a useful research tool to evaluate the immunogenicity of anthrax vaccines in humans.

\section{REFERENCES}

Adone, R., and Ciuchini, F. (1999). Complement fixation test to asses humoral immunity in cattle and sheep vaccinated with Brucella abortus RB51. Clin. Diagn. Lab. Immunol. 6, 787-790.

Adone, R., Francia, M., and Ciuchini, F. (2008). Brucella melitensis B115based complement fixation test to detect antibodies induced by Brucella rough strains. J. Appl. Microbiol. 105, 567-574. doi: 10.1111/j.1365-2672.2008. 03787.x

Beedham, R. J., Turnbull, P. C. B., and Williamson, E. D. (2001). Passive transfer of protection against Bacillus anthracis infection in a murine model. Vaccine 19, 4409-4416. doi: 10.1016/S0264-410X(01)00197-9

Biswas, P. K., Islam, M. J., Shil, S. K., Chakraborty, R. K., Ahmed, S. S., and Christensen, J. P. (2011). Risk factors associated with anthrax in cattle on smallholdings. Epidemiol. Infect. 140, 1888-1895. doi: $10.1017 /$ S0950268811002408

Centers for Diseases Control and Prevention, Division of Foodborne, Bacterial and Mycotic Diseases (2009). Anthrax Sterne Strain (34F2) of Bacillus anthracis. Available at: http://www.cdc.gov/nczved/divisions/dfbmd/diseases/ anthrax_sterne

Cho, H. J. (1971). Demonstration of complement fixing antibody in the sera of cattle vaccinated with combined living blackleg-anthrax vaccine. Can. J. Comp. Med. 35, 155-160.

Collier, R. J., and Young, J. A. (2003). Anthrax toxin. Annu. Rev. Cell Dev. Biol. 19, 45-70. doi: 10.1146/annurev.cellbio.19.111301.140655
This methodology, if officially accepted, could replace the lethal challenge with virulent anthrax strain, which is an expensive and increasingly unacceptable test.

The Sterne-based CFT could be particularly useful in veterinary medicine since serological tests are mostly involved in testing of herds or flocks of animals rather than single animals; the analysis of serological data should indicate whether the herd/flock is infected/vaccinated or not to obtain epidemiological information.

The CFT results indicated that the production of specific antibodies induced by vaccination with Sterne $34 \mathrm{~F}_{2}$ did not persist over a long period of time in cattle. In many studies, conducted in cattle and goats, the antibody response following vaccination against anthrax was monitored using a PA-based ELISA, currently accepted as the best serological procedure, and different antibody kinetics were observed (Dipti et al., 2013; Roy et al., 2013; Hassan et al., 2015). However, the comparison of serological data is very difficult since many factors affect the immune response of animals, such as the vaccine type, the age of vaccination and, mostly, the health status of animals (IOWA Beef Center website, 2015).

The importance of antibody kinetics in relation to the vaccine efficacy has been well demonstrated; it is a crucial information that should be taken in consideration for the implementation of effective prevention and control measures to protect population, also considering that B. anthracis is one of the preferred pathogenic agents for use as bacteriological weapon in bioterrorism attacks (Inglesby et al., 1999).

\section{ACKNOWLEDGMENT}

Funding for this research was provided by Italian Ministry of Health (Project IZS PB 02/12/RC).

Dipti, M., Rashid, M. M., Ferdoush, M. J., Roy, P., Khan, M. A. H. N. A., and Hossain, M. M. (2013). Morphological and immunological characterization of anthrax vaccine in cattle. Bangladesh J. Vet. Med. 11, 43-49.

Fasanella, A., Fabiano, M. P., Garofolo, G., and Losito, S. (2007). "Preliminary studies on recombinant protective antigen ( $\mathrm{rPA}$ ) vaccine against anthrax for veterinary use," in Abstracts of the International Veterinary Vaccines and Diagnostic Conference, Oslo, 69.

Fasanella, A., Losito, S., Trotta, T., Adone, R., Massa, S., Ciuchini, F., et al. (2001). Detection of anthrax vaccine virulence factors by polymerase chain reaction. Vaccine 19, 4214-4218. doi: 10.1016/S0264-410X(01) 00159-1

Fasanella, A., Tonello, F., Garofolo, G., Muraro, L., Carattoli, A., Adone, R., et al. (2008). Protective activity and immunogenicity of two recombinant anthrax vaccines for veterinary use. Vaccine 26, 5684-5688. doi: 10.1016/j.vaccine.2008.08.026

Friedlander, A. M., Welkos, S. L., and Ivins, B. E. (2002). “Anthrax vaccines," in Current Topics in Microbiology and Immunology, ed. T. M. Koehler (Houston, TX: University of Texas Medical School), 33-60.

Ghosh, N., and Goel, A. K. (2012). Anti-protective IgG enzyme-linked immunosorbent assay for diagnosis of cutaneous anthrax in India. Clin. Vaccine Immunol. 19, 1238-1242. doi: 10.1128/CVI.00154-12

Hassan, J., Rahman, M. B., Chowdhury, S. M. Z. H., Rabidas, S. K., Parvej, M. S., and Nazir, K. H. M. N. H. (2015). ELISA based anthrax antibody titer in cattle induced by locally prepared anthrax vaccine originated from sterne F-24 strain in Bangladesh. Microbes Health 4, 36-38. 
Inglesby, T. V., Henderson, D. A., Barlett, J. G., Ascher, M. S., Eitzen, E., Friedlander, A. M., et al. (1999). Anthrax as a biological weapon: medical and public health management. J. Am. Med. Assoc. 281, 1735-1745. doi: 10.1001/jama.281.18.1735

IOWA Beef Center website (2015). Beef Cattle Handbook by Richard C. Bull. Available at: http://www.iowabeefcenter.org/Beef\%20Cattle\%20Handbook/ Trace_Minerals_Immunology.pdf [Accessed February 1, 2015].

Ivins, B. E., and Welkos, S. L. (1988). Recent advances in the development of an improved anthrax vaccine. Eur. J. Epidemiol. 4, 12-19. doi: 10.1007/BF00152686

Ivins, B. E., Welkos, S. L., Knudson, G. B., and Little, S. F. (1990). Immunization against anthrax with aromatic compound-dependent (Aro-) mutants of Bacillus anthracis and with recombinant strains of Bacillus subtilis that produce anthrax protective antigen. Infect. Immun. 58, 303-308.

Kobiler, D., Gozes, Y., Rosenberg, H., Marcus, D., Reuveny, S., and Altboum, Z. (2002). Efficiency of protection of guinea pigs against infection with Bacillus anthracis spores by passive immunization. Infect. Immunol. 70, 544-550. doi: 10.1128/IAI.70.2.544-550.2002

Little, S. F., Ivins, B. E., Fellows, P. F., and Friedlander, A. M. (1997). Passive protection by polyclonal antibodies against Bacillus anthracis infection in guinea pigs. Infect. Immun. 65, 5171-5175.

Little, S. F., Webster, W. M., Ivins, B. E., Fellows, P. F., Norris, S. L., and Andrews, G. P. (2004). Development of an in vitro-based potency assay for anthrax vaccine. Vaccine 22, 2843-2852. doi: 10.1016/j.vaccine.2003.12.027

Mongoh, M. N., Dyer, N. W., Stoltenow, C. L., Hearne, R., and Khaitsa, M. L. (2008). A review of management practices for the control of anthrax in animals: the 2005 anthrax epizootic in North Dakotacase study. Zoonoses Public Health 55, 279-290. doi: 10.1111/j.1863-2378.2008.01135.x

Office International des Epizooties [OIE] (2008). Manual of Diagnostic Tests and Vaccines for Terrestrial Animals, 6th Edn. Paris: Office International des Epizooties.

Pitt, M. L. M., Little, S. F., Ivins, B. E., Fellows, P., Barth, J., Hewetson, J., et al. (2001). In vitro correlate of immunity in a rabbit model of inhalational anthrax. Vaccine 19, 4768-4773. doi: 10.1016/S0264-410X(01)00234-1

Quinn, C. P., Dull, P. M., Semenova, V., Li, H., Crotty, S., Taylor, T. H., et al. (2004). Immune responses to Bacillus anthracis protective antigen in individuals with bioterrorism-associated cutaneous and inhalation anthrax. J. Infect. Dis. 190, 228-1236. doi: 10.1086/423937

Reuveny, S., White, M. D., Adar, Y. Y., Kafri, Y., Altboum, Z., Gozes, Y., et al. (2001). Search of correlates of protective immunity conferred by anthrax vaccine. Infect. Immunol. 69, 2888-2893. doi: 10.1128/IAI.69.5.2888-2893. 2001

Roy, P., Rashid, M. M., Ferdoush, M. J., Dipti, M., Chowdury, M. G. A., Mostofa, M. G., et al. (2013). Biochemical and immunological characterization of anthrax spore vaccine in goat. Bangladesh J. Vet. Med. 11, 151-157.

Sterne, M. (1939). The use of anthrax vaccines prepared from avirulent (uncapsulated) variants of Bacillus anthracis. Onderstepoort J. Vet. Sci. Anim. Industry 13, 307-312.

Sterne, M. (1959). “Anthrax," in Infectious Diseases of Animals, Vol. 1, eds A. W. Stableforth and I. A. Galloway (London: Butterworths), 16-52.

Turnbull, P. C., Doganay, M., Lindeque, P. M., Aygen, B., and McLaughlin, J. (1992). Serology and anthrax in humans, livestock and Etosha National Park wildlife. Epidemiol. Infect. 108, 299-313. doi: 10.1017/S0950268800049773

Turnbull, P. C., Leppla, S. H., Broster, M. G., Quinn, C. P., and Melling, J. (1988). Antibodies to anthrax toxin in humans and guinea-pigs and their relevance to protective immunity. Med. Microbiol. Immunol. 177, 293-303.

Turnbull, P. C., Tindall, B. W., Coetzee, J. D., Conradie, C. M., Bull, R. L., Lindeque, P. M., et al. (2004). Vaccine-induced protection against anthrax in cheetah (Acinonyxjubatus) and black rhinoceos (Dicerosbicornis). Vaccine 22, 3340-3347. doi: 10.1016/j.vaccine.2004.02.037

Welkos, S., Little, S., Friedlander, A., Fritz, D., and Fellows, P. (2001). The role of antibodies to Bacillus anthracis and anthrax toxin components in inhibiting the early stages of infection by anthrax spores. Microbiology 147, 1677-1685. doi: 10.1099/00221287-147-6-1677

World Health Organization [WHO] (2008). Anthrax in Animal and Humans, 4th Edn. Geneva: World Health Organization. Available at: http://www.who.int/csr/resources/publications/anthrax_webs.pdf

Conflict of Interest Statement: The authors declare that the research was conducted in the absence of any commercial or financial relationships that could be construed as a potential conflict of interest.

Copyright (C) 2016 Adone, Sali, Francia, Iatarola, Donatiello and Fasanella. This is an open-access article distributed under the terms of the Creative Commons Attribution License (CC BY). The use, distribution or reproduction in other forums is permitted, provided the original author(s) or licensor are credited and that the original publication in this journal is cited, in accordance with accepted academic practice. No use, distribution or reproduction is permitted which does not comply with these terms. 